Supporting Information For:

\title{
PEGylated nanoscale metal-organic frameworks for targeted cancer imaging and drug delivery
}

Yawen Zeng ${ }^{\mathrm{a}, 1}$, Jinling Xiao ${ }^{\mathrm{a}, 1}$, Yiyang Cong ${ }^{\mathrm{b}}$, Jia Liu ${ }^{\mathrm{a}}$, Yiming He $\mathrm{He}^{\mathrm{a}}$, Brian D. Ross ${ }^{\mathrm{c}}$, Haixing $\mathrm{Xu}^{\mathrm{a}}$, Yihua Yin ${ }^{\mathrm{a}}$, Hao Hong ${ }^{\mathrm{b}, * *}$, Wenjin $\mathrm{Xu}^{\mathrm{a}, *}$

${ }^{\text {a }}$ Department of Pharmaceutical Engineering, School of Chemistry, Chemical Engineering and Life Sciences, Wuhan University of Technology, 122 Luoshi Road, Wuhan,Hubei, 430070, China

${ }^{\mathrm{b}}$ Medical School of Nanjing University, 22 Hankou Rd, Nanjing, Jiangsu 210093, China.

c Center for Molecular Imaging, Department of Radiology, 109 Zina Pitcher Place, Ann Arbor, MI 48109-2200, USA.

* To whom correspondence should be addressed:

*Corresponding author: Department of Pharmaceutical Engineering, School of Chemistry, Chemical Engineering and Life Sciences, Wuhan University of Technology, 122 Luoshi Road, Wuhan, Hubei, 430070, China

** Corresponding author: Jiangsu Key Laboratory of Molecular Medicine, Medical School \& Chemistry and Biomedicine Innovation Center of Nanjing University, 22 Hankou Rd, Nanjing, Jiangsu 210093, China. 
Email addresses: chemxu@aliyun.com (W. Xu), haohong@nju.edu.cn (H. Hong)

${ }^{1}$ These two authors contributed equally.

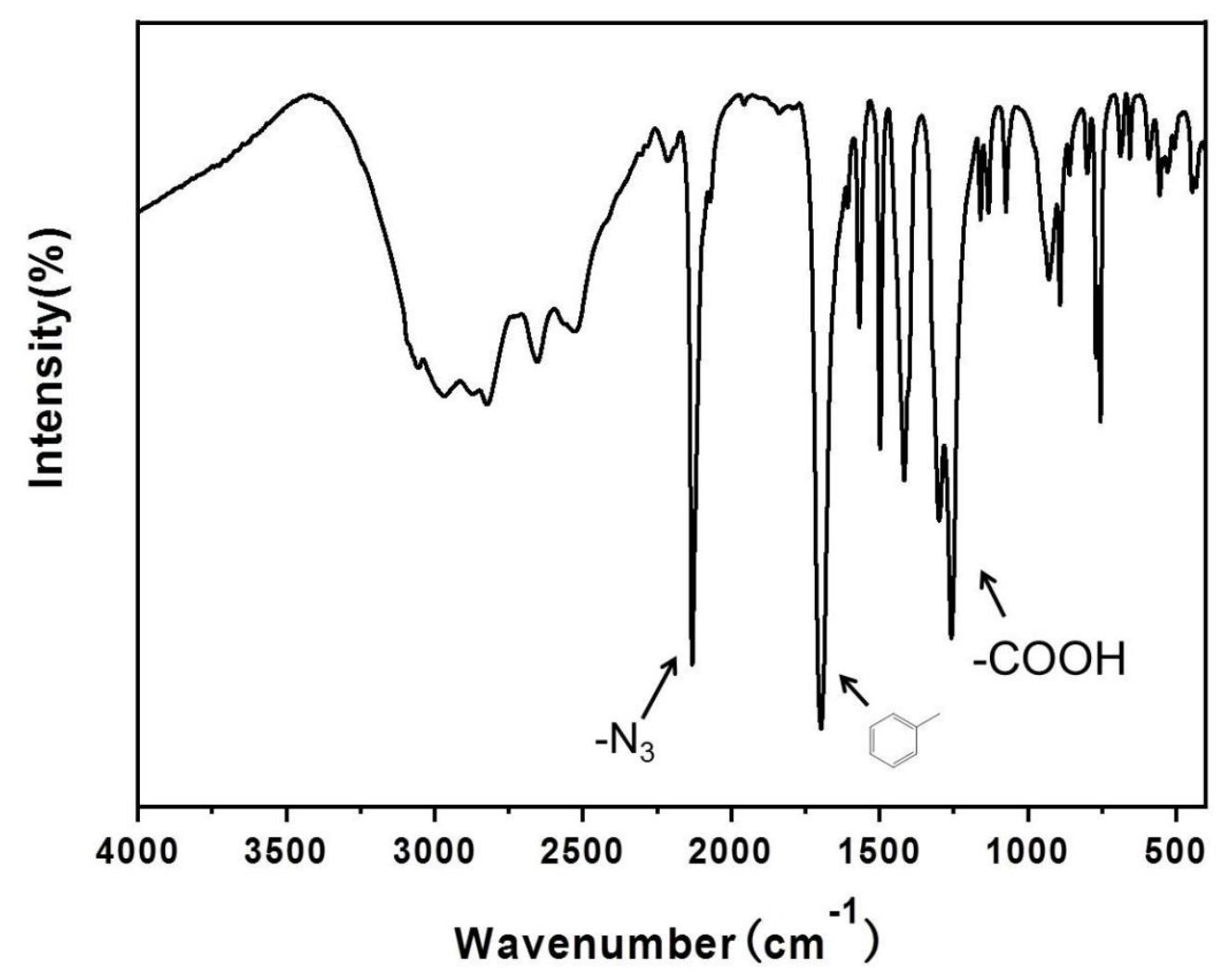

Figure S1. FT-IR spectrum of BDC-N $\mathrm{N}_{3}$. 


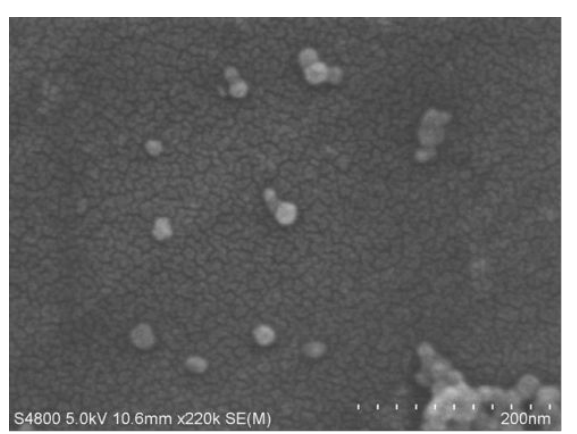

UiO-66-N3

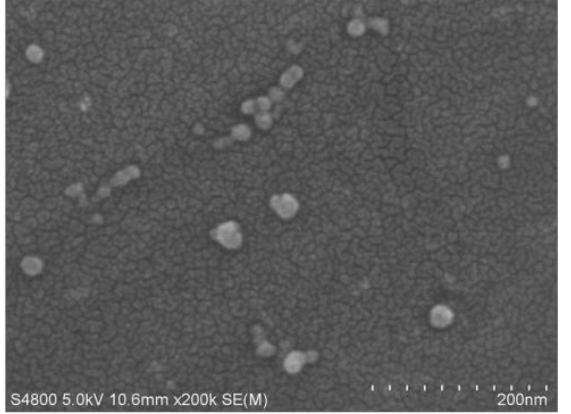

UiO-66-PEG

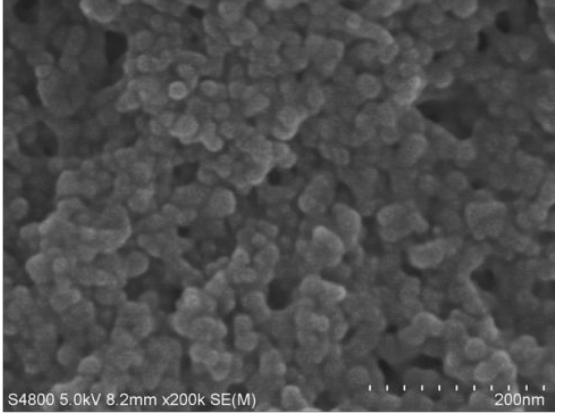

UiO-66-PEG-F3

Figure S2. SEM images of (a) UiO-66-N 3 , (b) UiO-66-PEG, and (c) UiO-66-PEG-F3. Scale bar: $200 \mathrm{~nm}$.

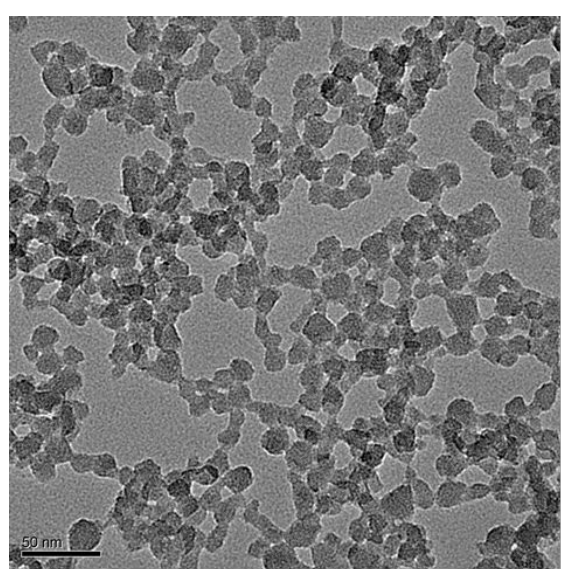

UiO-66-N3

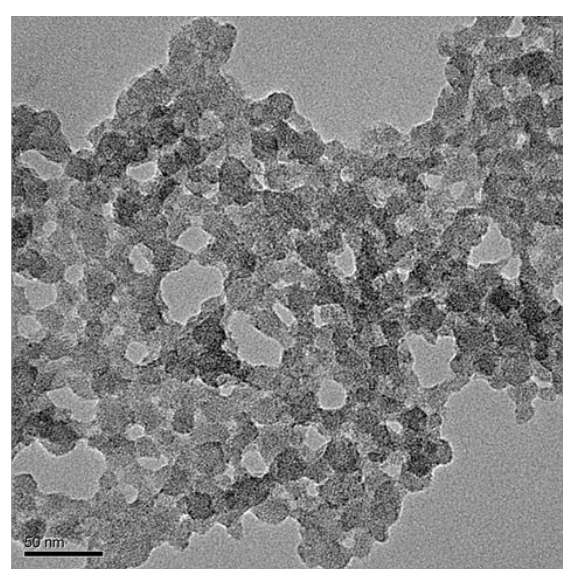

UiO-66-PEG

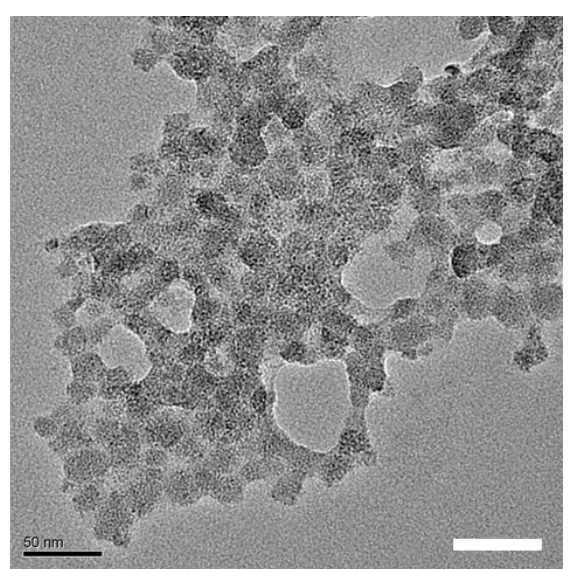

UiO-66-PEG-F3

Figure S3. TEM images of (a) UiO-66- $\mathrm{N}_{3}$, (b) UiO-66-PEG, and (c) UiO-66-PEG-F3. Scale bar: $50 \mathrm{~nm}$. 


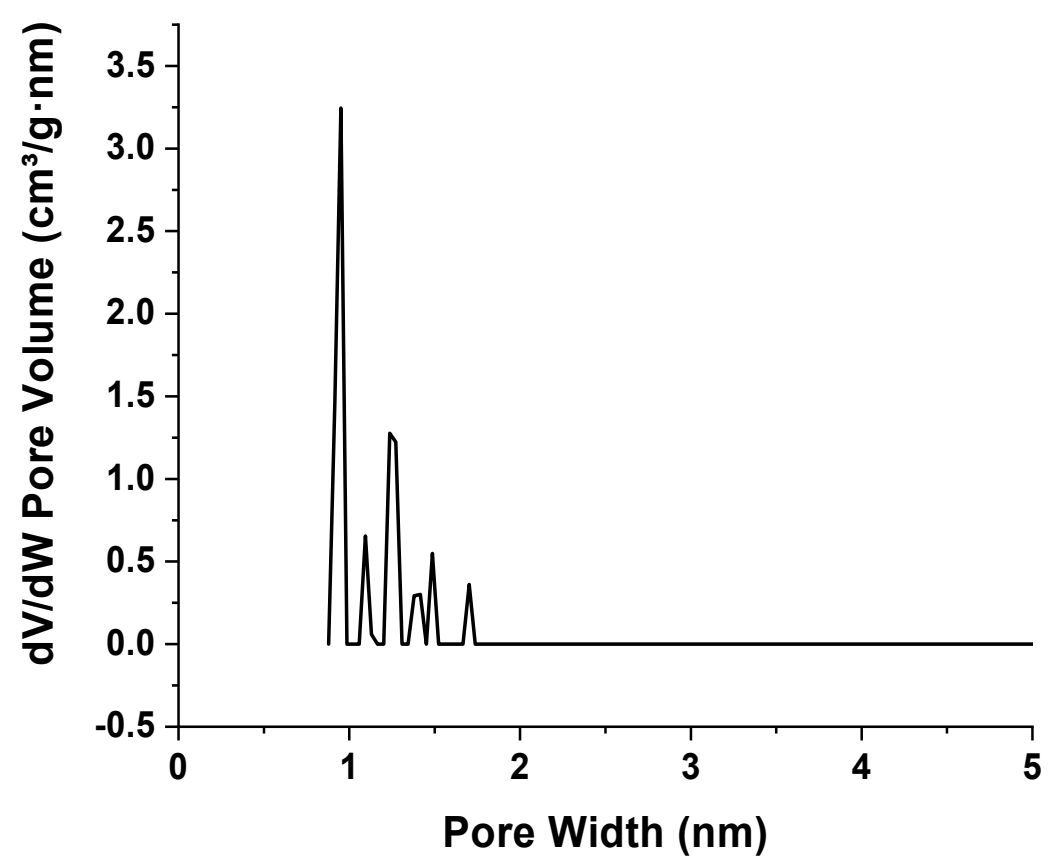

Figure S4. Pore size distributions of UiO-66- $\mathrm{N}_{3}$ determined from nitrogen uptake measurements.

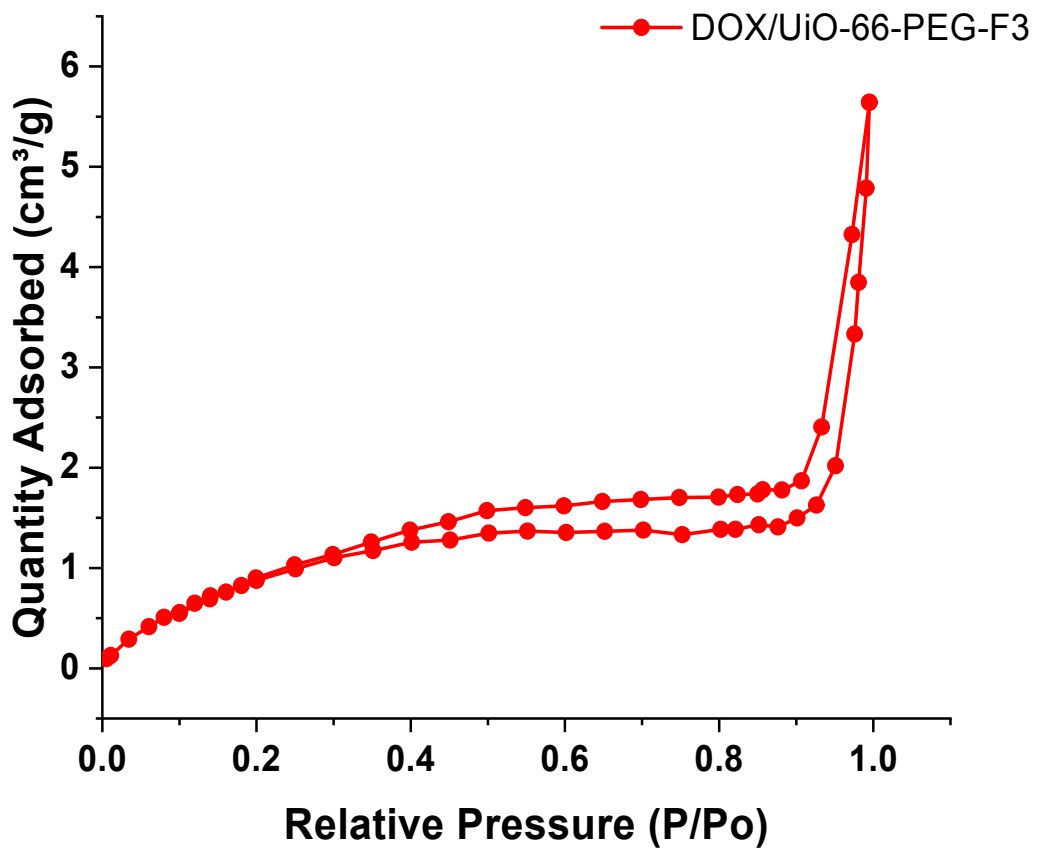

Figure S5. Nitrogen absorption curves of DOX/UiO-66-PEG-F3. 


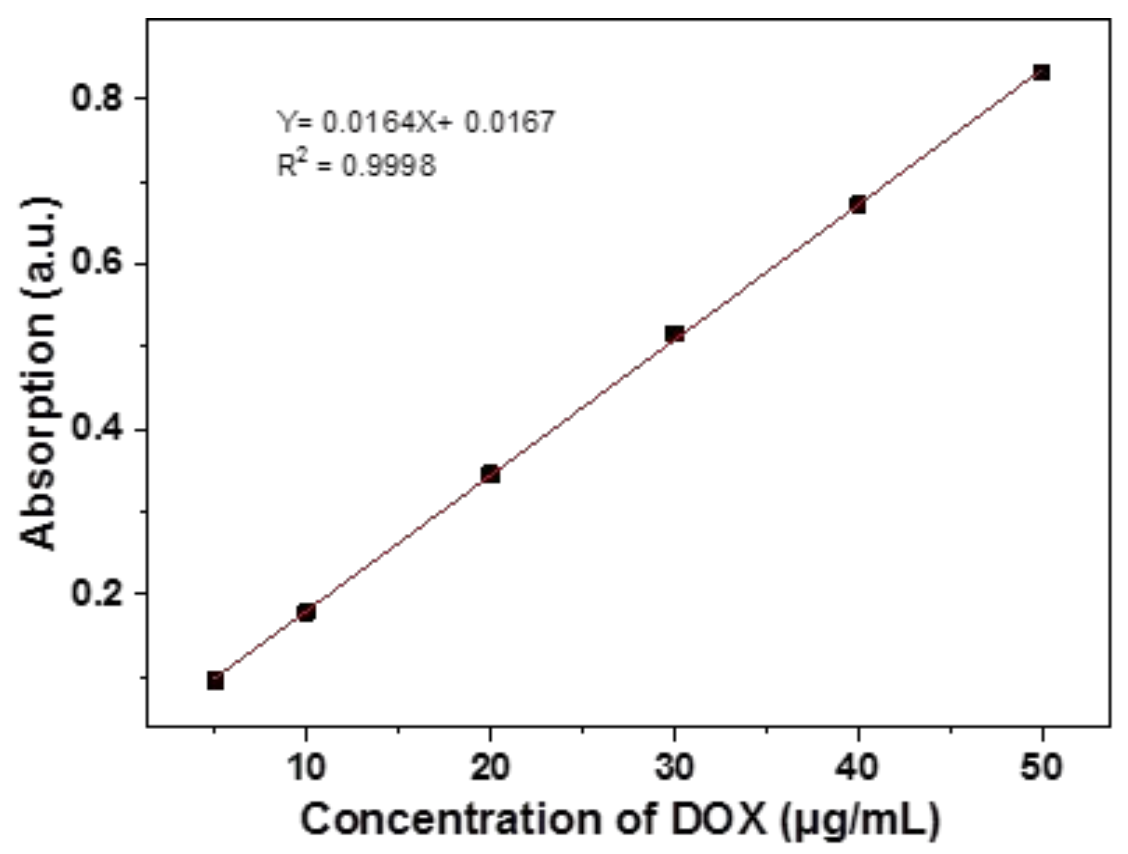

Figure S6. Standard curve of DOX based on UV-Vis measurement.

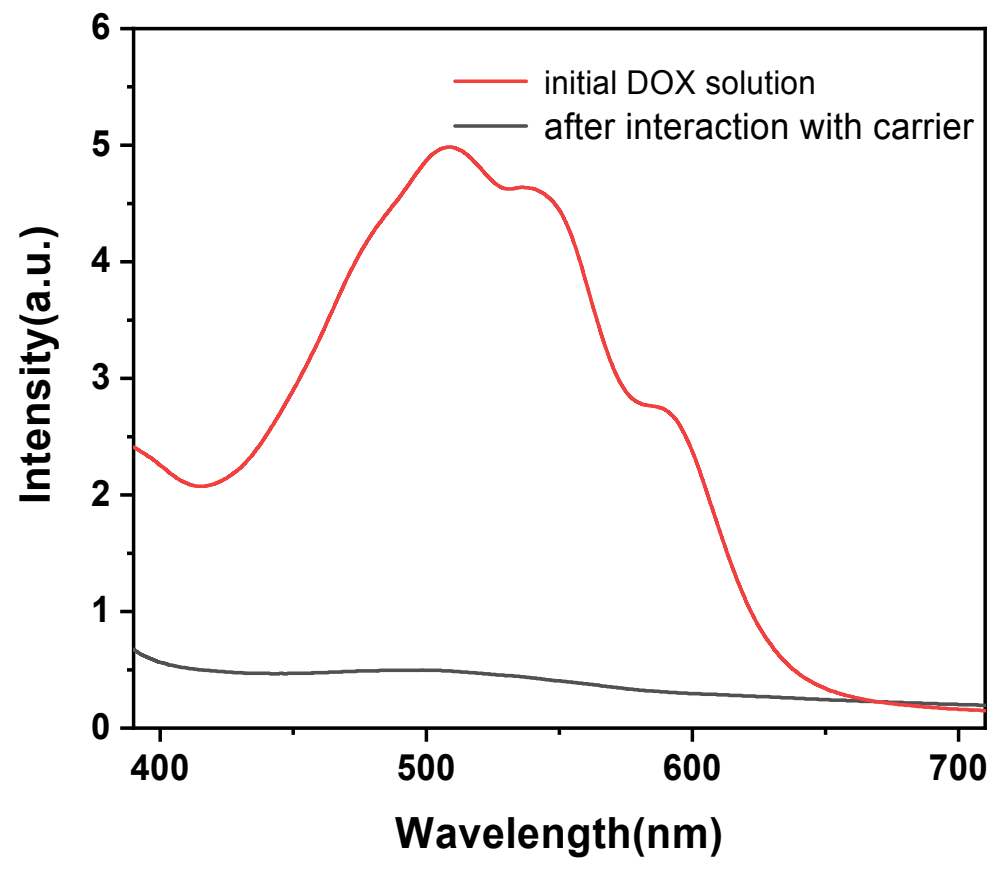


Figure S7. UV-Vis spectroscopy of free DOX and DOX after loading.

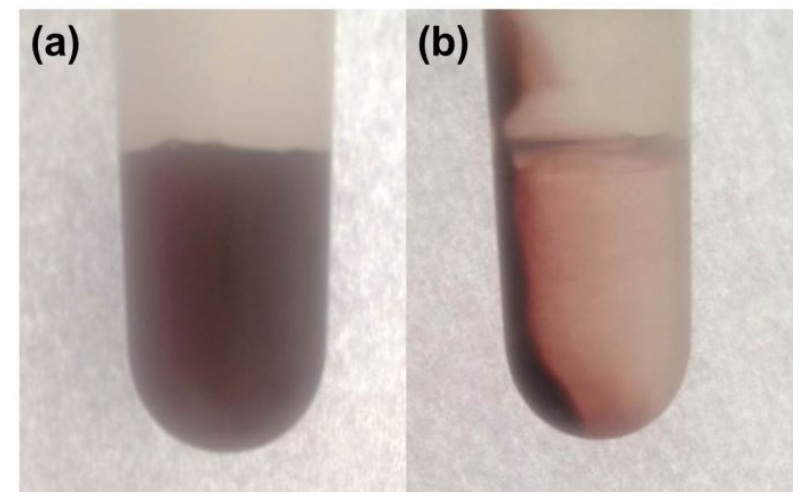

Figure S8. Camera photo image of (a) free DOX and (b) DOX after loading into UiO-66- $\mathrm{N}_{3}$.

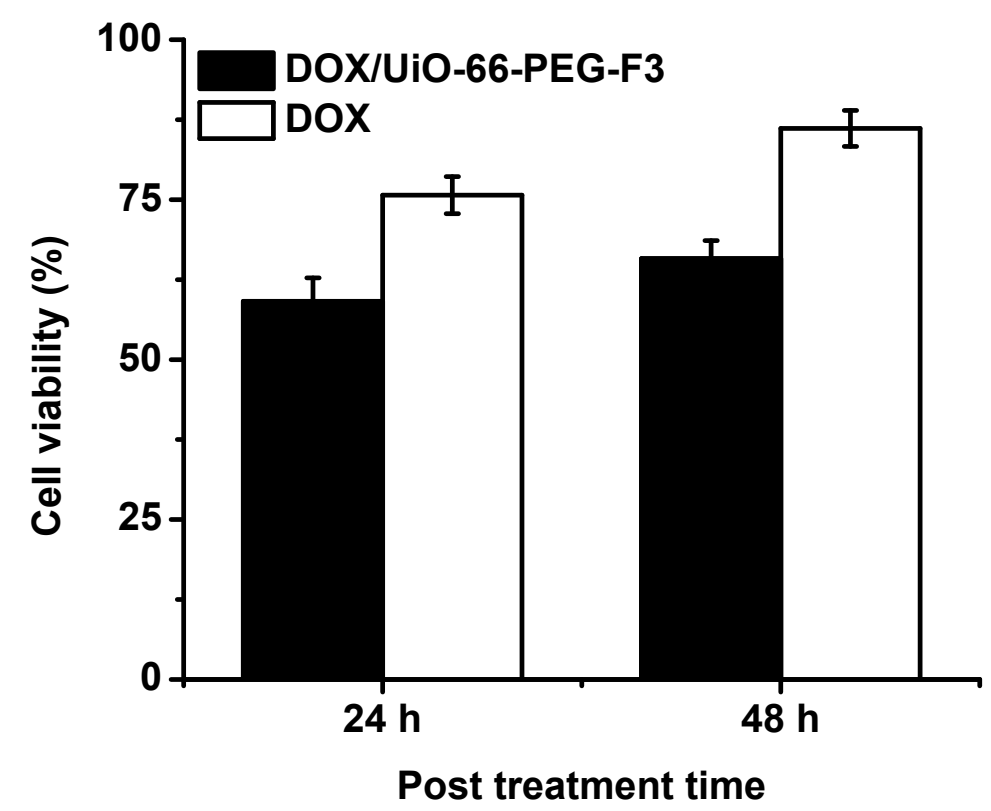

Figure S9. Persistent (24 h and 48 h post treatment) cytotoxicity of DOX and DOX@UiO-66PEG-F3 to MDA-MB-231 cells 


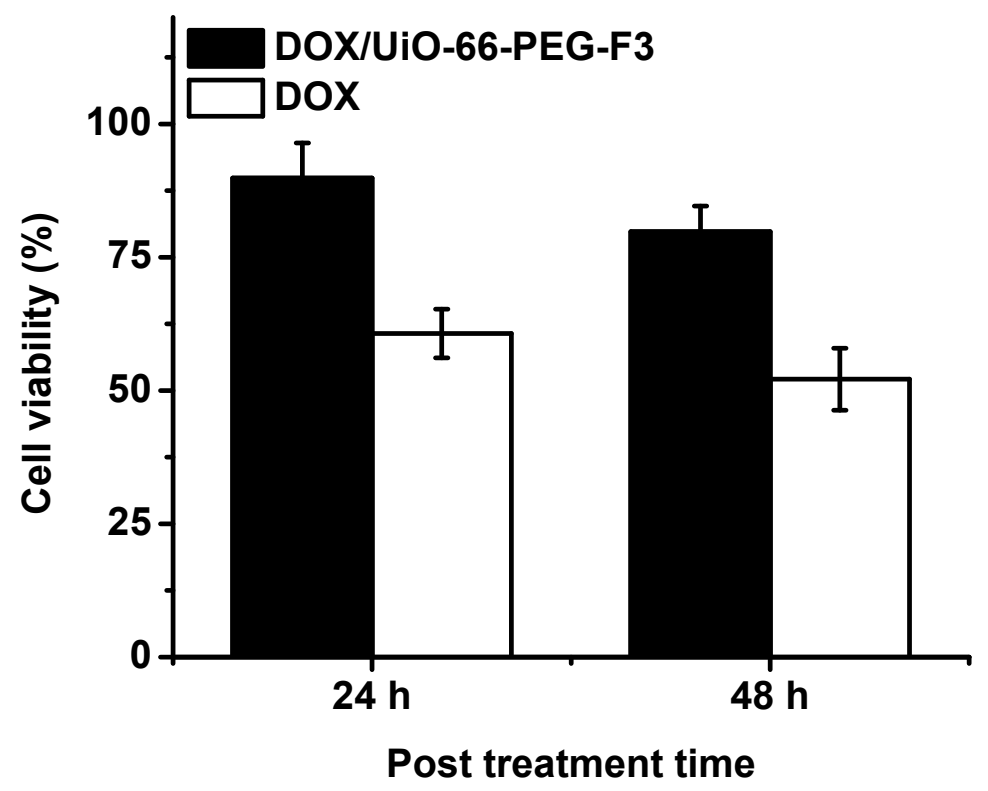

Figure S10 The cytotoxicity of DOX and DOX@UiO-66-PEG-F3 to L929 cells (24 h and 48 h treatment).

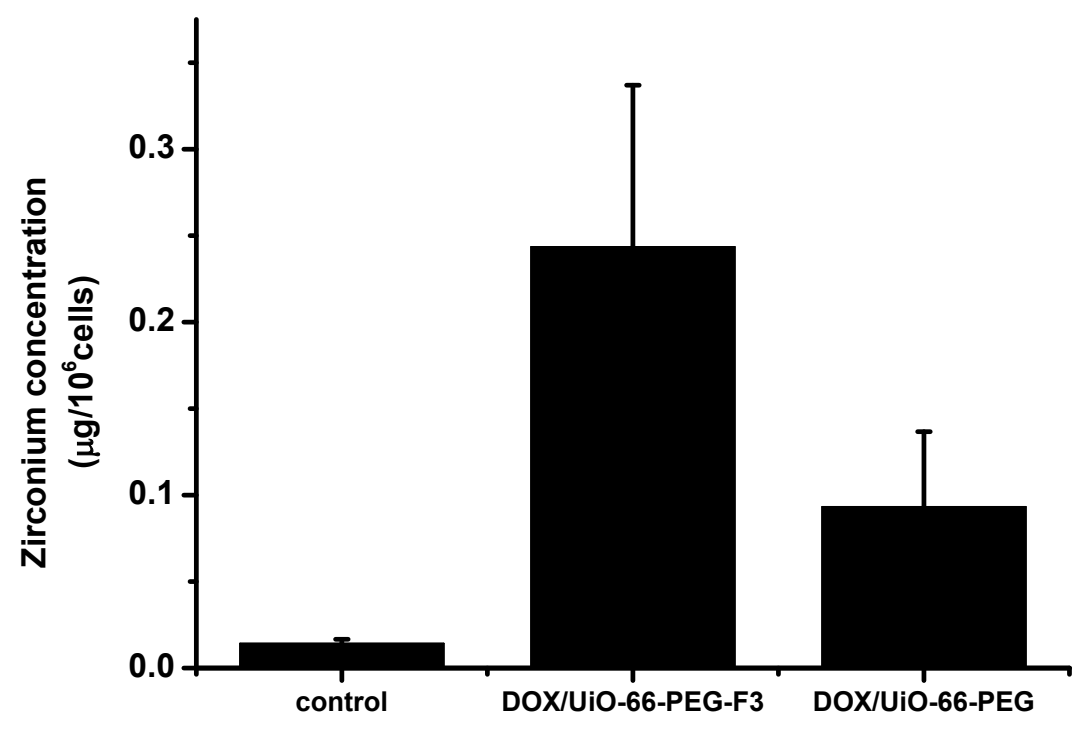

Figure S11 ICP-MS analysis of zirconium concentration inside the MDA-MB-231 cells post incubation with DOX/UiO-66-PEG-F3 and DOX/UiO-66-PEG. 
Table S1. The Z-Average and Polymer dispersity index of UiO-66- $\mathrm{N}_{3}$, UiO-66-PEG, UiO-66PEG-F3 and DOX/UiO-66-PEG-F3

\begin{tabular}{ccc}
\hline Sample & Z-Average (d.nm) & Polymerdispersity index(PDI) \\
\hline UiO-66-N 3 & 124.2 & 0.080 \\
UiO-66-PEG & 161.1 & 0.097 \\
UiO-66-PEG-F3 & 172.7 & 0.090 \\
DOX/UiO-66-PEG-F3 & 201.0 & 0.120 \\
\hline
\end{tabular}

Table S2. The Zeta potential and mobility values of UiO-66- $\mathrm{N}_{3}$, UiO-66-PEG, UiO-66-PEG-F3 and DOX/UiO-66-PEG-F3

\begin{tabular}{ccc}
\hline Sample & Zeta potential (mv) & Mobility $(\boldsymbol{\mu m c m} / \mathbf{V s})$ \\
\hline UiO-66-N 3 & $-23.6 \pm 0.5$ & -0.9622 \\
UiO-66-PEG & $-12.2 \pm 0.5$ & -0.7958 \\
UiO-66-PEG-F3 & $-10.7 \pm 0.5$ & -0.7187 \\
DOX/UiO-66-PEG-F3 & $-0.224 \pm 0.5$ & -0.2979 \\
\hline
\end{tabular}

